\title{
Comparação entre protocolos de tratamento para a estefanofilariose em vacas leiteiras ${ }^{1}$
}

\section{Comparison among therapeutic protocols for stephanofilariasis in dairy cows}

\author{
Vanessa Issuzu Miyakawa²; Antonio Carlos Faria dos Reis ${ }^{3}$; \\ Júlio Augusto Naylor Lisbôa ${ }^{4}$
}

\section{Resumo}

\begin{abstract}
A estefanofilariose, causada por um nematódeo do gênero Stephanofilaria e transmitida por moscas, é mais prevalente no verão e caracteriza-se por uma dermatite crônica localizada no úbere de vacas leiteiras. Algumas alternativas de tratamento já foram testadas e os resultados relatados são variáveis. O objetivo do trabalho foi comparar a eficácia de diferentes tratamentos para a doença. Vacas leiteiras naturalmente acometidas por estefanofilariose e exibindo feridas características no úbere foram distribuídas por quatro grupos de tratamento tópico: G1 ( $\mathrm{n}=11)$ uso de ivermectina $1 \%$ em pasta; $\mathrm{G} 2(\mathrm{n}=10)$ uso de ivermectina $2 \%$ em pasta; G3 ( $\mathrm{n}=10)$ uso de triclorfon $6 \%$ em pasta de vaselina; e G4 $(\mathrm{n}=11)$ o mesmo tratamento empregado no G3 mais ivermectina pour-on em dose única ou repetida a cada 42 dias. As pastas foram aplicadas nas feridas duas vezes ao dia até a cura completa. A ferida foi mensurada semanalmente desde o dia inicial do tratamento até a sua cicatrização completa. Não houve diferenças entre os tratamentos considerando a área original da ferida $(p=0,820)$, o tempo de duração do tratamento até a cura $(p=$ $0,168)$ e a redução diária da área da ferida $(p=0,817)$. Todos os tratamentos foram eficazes permitindo que a cura fosse alcançada em todos os animais. O tratamento com triclorfon $6 \%$ em pasta de vaselina foi o mais vantajoso porque apresentou a melhor relação custo-benefício.
\end{abstract}

Palavras-chave: Stephanofilaria sp., bovines, triclorfon, ivermectina

\begin{abstract}
Stephanofilariaisis is a disease caused by the nematode Stephanofilaria, transmitted by flies, that determines a chronic dermatitis in the udder of lactating cows. The prevalence is higher in summer. Different treatment options have already been tested with varying results. The aim of this work was compare the efficacy of four topical treatment protocols. Naturally affected dairy cows suffering from characteristic udder skin lesion were distributed by four groups of treatment: G1 ( $\mathrm{n}=11) 1 \%$ ivermectin; G2 $(n=10) 2 \%$ ivermectin; G3 $(n=10) 6 \%$ trichlorfon; and $\mathrm{G} 4(\mathrm{n}=11) 6 \%$ trichlorfon plus pour on ivermectin. The ointments were applied in the wounds twice a day until the complete healing. The pour on ivermectin was applied once in the beginning of the treatment and repeated 42 days late. The wound area was measured before the start of the therapy and every seven days until the complete recovery. There were no treatment differences considering the initial area of the wound $(p=0,820)$, the length of time required to recovery $(p=0,168)$, and the daily decrease in the area of the wound $(p=0,817)$. All four treatment protocols tested were successful and the outcome was reached in all cows. The treatment with $6 \%$ trichlorfon in vaseline oitment was more profitable showing the better cost-benefit relation.
\end{abstract}

Key words: Stephanofilaria sp., cattle, trichlorfon, ivermectin

${ }^{1}$ Esse trabalho é parte da Dissertação do primeiro autor.

2 Mestre pelo Programa de Pós-graduação em Ciência Animal, Universidade Estadual de Londrina, UEL. Londrina, PR. E-mail: issuzu@gmail.com

3 Prof. Dr. do Dept ${ }^{\circ}$ de Medicina Veterinária Preventiva, UEL. Londrina, PR. E-mail: reis@uel.br

${ }^{4}$ Prof. Dr. do Dept ${ }^{\circ}$ de Clínicas Veterinárias, UEL. Londrina, PR. E-mail: janlisboa@uel.br

Autor para correspondência 


\section{Introdução}

A estefanofilariose caracteriza-se por lesões na pele causadas por um nematódeo do gênero Stephanofilaria. O parasita foi primeiramente descrito em lesões de bovinos na Indonésia e relatos frequentes foram reportados no Canadá, EUA, Rússia, Índia, Malásia, Japão e países da África (JOHNSON et al., 1981). No Brasil, o primeiro caso foi relatado no Estado de São Paulo por Birgel et al. 1972.

Quanto à localização das feridas nos bovinos, elas já foram observadas próximas à cauda, na garupa, na coxa(NOVAES et al., 1988) e na quartela (NOVAES; OLIVEIRA; MOREIRA, 1990). As lesões são frequentes na pele da mama, especialmente, na porção anterior do úbere $\mathrm{e}$ acometem preferencialmente as vacas lactantes (BIRGEL et al., 1972; SILVA; BRAGA; FIORAVANTE, 2001; SILVA et al., 2010; MIYAKAWA; REIS; LISBÔA, 2009). A lesão cutânea caracteriza-se inicialmente por uma erupção papular progredindo para nódulo, alopecia e úlcera crostosa. O prurido é comum e, na maioria dos casos, uma secreção serossanguinolenta é observada na ferida o que atrai moscas, consideradas hospedeiros intermediários e vetores da doença. A lesão, única ou não, pode variar em tamanho e permanece presente por um longo tempo se não tratada, podendo persistir por dois a três anos (SCOTT, 1988).

O tratamento é importante não somente por questões estéticas, mas principalmente para eliminar o incômodo relacionado ao prurido, evitar complicações possíveis como miíases e mastite, além de impedir a infecção do hospedeiro intermediário, diminuindo a incidência da parasitose no rebanho (PATNAIK, 1970; MIYAKAWA; REIS; LISBÔA, 2009).

Há poucos estudos sobre o tratamento dessa doença em bovinos. Alguns autores recomendam o triclorfon tópico (SRIVASTAVA; MALVIYA, 1968; PATNAIK, 1970; NOVAES et al., 1988) ou por via subcutânea (OBA et al., 1977), a ivermectina (GILL et al., 1991) e o levamisol (RAI et al., 1994) por via parenteral, e ainda a associação do triclorfon tópico e da ivermectina injetável (SILVA; BRAGA; FIORAVANTE, 2001; SILVA et al., 2010). A eficácia do tratamento é variada dependendo do tempo de evolução e do tamanho da lesão e algumas dessas alternativas não podem ser adotadas para o tratamento de vacas durante a lactação, por causarem a presença de resíduos no leite. A eficiência de um tratamento tópico exclusivo com a ivermectina ainda não foi testada. Esse trabalho foi delineado com o objetivo de comparar a eficácia de diferentes protocolos para o tratamento de vacas leiteiras naturalmente acometidas por estefanofilariose.

\section{Material e Métodos}

Foram avaliados e acompanhados, de janeiro de 2006 a agosto de 2008, 58 casos naturais de estefanofilariose em vacas pertencentes a sete propriedades produtoras de leite localizadas nos municípios de Santana do Itararé, PR e de Itaberá, SP, distribuídas num raio aproximado de $60 \mathrm{~km}$. Nesses rebanhos a doença era endêmica (MIYAKAWA; REIS; LISBÔA, 2009).

As propriedades acompanhadas possuíam rebanhos com características raciais semelhantes (HPB, Jersey e seus cruzamentos) e produção de média de 20 litros de leite diários por vaca. Adotavam padrão de manejo extensivo. O manejo nutricional consistia em pastejo rotacionado no verão (Panicum maximum cv. Mombaça e/ou Pennicetum purpureum cv. Pioneiro) e em ingestão de silagem de milho e/ ou sorgo, além de cana-de-açúcar com uréia no inverno. Mesmo nos meses de baixa precipitação pluviométrica os animais eram alimentados nos piquetes (rotação na área de piquetes para adubação natural). A ordenha mecanizada era realizada duas vezes ao dia, ocasiões em que o concentrado era oferecido em quantidade variável de acordo com a produção de leite individual.

As vermifugações eram mais frequentes nas bezerras, as quais recebiam dois tratamentos até os 
60 dias de vida e mais três doses até a puberdade. Os princípios anti-helmínticos utilizados alternadamente eram a ivermectina, o albendazol, o levamisol e a moxidectina. As vacas eram vermifugadas somente no momento da secagem, realizada 60 dias antes do parto, e não recebiam vermífugos durante todo o período de lactação.

Foram selecionadas para o estudo 42 vacas leiteiras portadoras de lesões características da doença localizadas na pele da mama. Com a finalidade de confirmação do diagnóstico, realizouse, em 24 vacas e previamente ao início do tratamento, colheita de amostra de tecido adjacente à borda da lesão cutânea por meio de biópsia com auxílio de um punch (6 mm de diâmetro interno). Tal procedimento foi precedido pela limpeza prévia do local com álcool $70 \%$ e anestesia local infiltrativa com $0,5 \mathrm{~mL}$ de lidocaína $2 \%$ (Lidovet $^{\circledR}$; Laboratório Bravet Ltda.). O fragmento era constituído de aproximadamente três partes do tecido aparentemente sadio e uma parte do tecido lesionado, com formato cilíndrico e tamanho aproximado de $6 \mathrm{~mm}$ de diâmetro e $10 \mathrm{~mm}$ de profundidade. Empregou-se o método preconizado por Ueno e Gonçalves (1998), o qual se baseia na migração dos parasitas do tecido para a solução no qual este fica embebido. O fragmento tecidual foi mantido submerso em solução fisiológica $(\mathrm{NaCl}$ $0,9 \%$ - 10 partes de solução para uma de tecido - em um recipiente plástico por, no mínimo, 3 horas em temperatura ambiente. Após a remoção do fragmento toda a solução do recipiente foi centrifugada (1.500 rpm por 5 minutos). Várias gotas do sedimento foram examinadas, entre lâmina e lamínula, ao microscópio óptico (aumento total de 100x: objetiva de 10x e ocular de 10x), comprovando a presença das formas adultas, e raramente larvares, da Stephanofilaria sp. (MIYAKAWA; REIS; LISBÔA, 2009).

A mensuração da ferida foi realizada com paquímetro, no primeiro exame físico e repetida semanalmente. Por se tratarem, na sua grande maioria, de feridas com aspecto circular, a área da ferida foi calculada aplicando-se a fórmula: área $\left(\mathrm{cm}^{2}\right)=\pi \cdot \mathrm{r}^{2}$; sendo $\mathrm{r}$ o raio (metade do diâmetro mensurado). Nas lesões com formato elíptico obtiveram-se duas medidas: o maior e o menor eixo; e a área foi calculada aplicando-se a fórmula: área $\left(\mathrm{cm}^{2}\right)=\pi$. a . b; sendo a e b os semi-eixos (metade do eixo mensurado) maior e menor, respectivamente.

Para efeito de comparação de sua eficácia, foram compostos quatro grupos de tratamento. No $\mathrm{G} 1(\mathrm{n}=11)$ as vacas receberam ivermectina $1 \%$ na forma de pasta (Ivermic Equinos ${ }^{\circledR}$; Laboratórios Microsules do Brasil) aplicada sobre a ferida duas vezes ao dia até a sua reepitelização completa. No $\mathrm{G} 2(\mathrm{n}=10)$ as vacas foram tratadas com ivermectina $2 \%$ em pasta $\left(\right.$ Altec $^{\circledR}$; Tortuga Cia. Zootécnica Agrária) aplicada de forma igualmente à descrita para o G1. No G3 (n=10) utilizou-se uma pomada preparada de vaselina em pasta veiculando triclorfon (Neguvon $^{\circledR}$; Bayer S.A.) na concentração de $6 \%$, aplicada de forma igual à descrita para o G1. No $\mathrm{G} 4(\mathrm{n}=11)$ as vacas receberam o mesmo tratamento do G3 mais a ivermectina pour-on (Supramec Pouron $^{\circledR}$; Schering-Plough Coopers) na dose de $0,5 \mathrm{mg} /$ $\mathrm{kg}$ de PV em aplicação única. Nos animais do G4 que necessitaram tratamento mais prolongado, uma segunda administração do produto pour-on foi realizada 42 dias após a primeira. As aplicações das pastas ou pomada sobre a ferida foram realizadas ao término dos procedimentos diários da ordenha. Quando havia muita secreção e sujidades realizavase uma limpeza do local com água corrente e secagem com papel toalha anteriormente ao tratamento. Aplicava-se quantidade de pasta ou de pomada suficiente para produzir uma cobertura completa da lesão cutânea, o que equivalia a aproximadamente $0,5 \mathrm{~g}$ no início do tratamento. Ao longo de todo o período do tratamento, as vacas foram acompanhadas com exames semanais para observação da lesão cutânea, avaliando seu aspecto e complicações, e para realização das mensurações.

As vacas tratadas possuíam lesões cutâneas únicas cujas áreas originais variavam entre 3 e 22 $\mathrm{cm}^{2}$, com tempo de evolução entre 6 e 25 dias. A 
distribuição das vacas pelos grupos de tratamento não foi completamente ao acaso porque procurouse manter uma proporcionalidade entre os grupos quanto à área inicial da ferida, fator que poderia exercer a maior influência nos resultados. O tempo de evolução da doença, a raça e o mês do ano em que se iniciou o tratamento foram outros fatores que acabaram distribuídos de maneira quase proporcional entre os grupos.

Empregou-se a análise de variância para a comparação entre os tratamentos, admitindo-se uma probabilidade de erro de $5 \%$. O coeficiente de correlação entre a área inicial da ferida e o total de dias necessários para a sua cicatrização foi determinado.

\section{Resultados e Discussão}

$\mathrm{Na}$ população estudada a estefanofilariose apresentou maior prevalência no verão, acometendo sobretudo as vacas lactantes sem predileção por idade, por raça ou pela fase da lactação. Caracterizouse pelo aparecimento de uma lesão cutânea única de formato circular ou elíptico, ulcerada com crosta e exsudato serossanguinolento, localizada no aspecto cranial do úbere (MIYAKAWA; REIS; LISBÔA, 2009).

Os quatro protocolos de tratamento adotados foram eficazes para promover a cura de forma equivalente, ou seja, sem nenhuma superioridade particular (Tab. 1). Não houve diferenças entre os grupos quanto à área inicial da lesão cutânea $(p=0,820)$, ao tempo total de duração do tratamento $(p=0,168)$ e à redução diária calculada da área da ferida $(p=0,817)$. Correlação $(\mathrm{r}=0,571 ; p<0,001)$ entre a área original da ferida e o tempo de duração do tratamento indica que quanto maior a área original da ferida, maior o número de dias necessários para a realização do tratamento até que a cicatrização fosse alcançada.

Os tratamentos se estenderam por um período de tempo relativamente longo. Considerando-se a média, prolongaram-se entre um e dois meses (Tabela 1). Duas vacas, entretanto, necessitaram de tratamento durante 11 (uma vaca no G4) a 12 (uma vaca no G1) semanas seguidas. Nessas vacas a área da lesão original era maior $\left(22 \mathrm{~cm}^{2}\right.$ e 20,8 $\mathrm{cm}^{2}$, respectivamente). No G2 e G3 a maioria das vacas se curou em até 8 semanas. Esses resultados são coerentes com os relatos de tratamentos realizados em rebanhos leiteiros no Estado de Goiás (SILVA; BRAGA; FIORAVANTE, 2001; SILVA et al., 2010). Duração de tratamento muito menor foi apontada em outros estudos, desenvolvidos na Índia, que utilizaram o triclorfon 6\% em aplicação tópica (PATNAIK, 1970; SRIVASTAVA; MALVIYA, 1968), embora Rahman e Khaleque (1974) tenham relatado que no caso de algumas feridas mais extensas o tempo necessário para a cura poderia ultrapassar 40 dias. Em todos esses estudos indianos a Stephanofilaria assamensis, espécie comumente encontrada no continente asiático, era o agente causador da doença. Ainda que não se conheça em definitivo que espécie do agente existe no Brasil, diferença entre as espécies de Stephanofilaria presentes nos dois países poderia, supostamente, explicar a distinção nos resultados dos tratamentos.

Tabela 1. Resultados $\left(\overline{\mathrm{x}}_{ \pm} \mathrm{s}\right)$ de diferentes protocolos empregados para o tratamento tópico de vacas leiteiras naturalmente acometidas por estefanofilariose.

\begin{tabular}{lccc}
\hline Tratamento & $\begin{array}{c}\text { Área inicial da ferida } \\
\left(\mathbf{( c m}^{\mathbf{2}}\right)\end{array}$ & $\begin{array}{c}\text { Tempo para cicatrizar } \\
(\mathbf{d i a s})\end{array}$ & $\begin{array}{c}\text { Redução diária da área } \\
\mathbf{d a} \text { ferida } \\
\left(\mathbf{c m}^{2} / \mathbf{d i a}\right)\end{array}$ \\
\hline Ivermectina 1\% & $9,12 \pm 6,73$ & $47,0 \pm 18,2$ & $0,18 \pm 0,11$ \\
Ivermectina 2\% & $7,20 \pm 3,24$ & $34,5 \pm 11,8$ & $0,21 \pm 0,07$ \\
Triclorfon 6\% & $9,20 \pm 5,23$ & $39,1 \pm 9,2$ & $0,23 \pm 0,12$ \\
Triclorfon 6\% + Ivermectina pour on & $9,40 \pm 7,30$ & $42,4 \pm 9,5$ & $0,20 \pm 0,13$ \\
\hline
\end{tabular}


No Brasil, há um relato do tratamento com triclorfon promover a cura em 15 dias (NOVAES et al., 1988). Contudo, esse estudo se resumiu à observação de cinco bovinos os quais receberam, além do triclorfon, a aplicação de repelentes, em forma de aerosol ou pó, na ferida. Os autores não especificaram os princípios ativos dos repelentes e a concentração do triclorfon utilizado. Em outra experiência, o tratamento semanal com triclorfon injetável teve que se prolongar por seis semanas para determinar a cura (OBA et al., 1977).

Aolongo do acompanhamento semanal observouse a redução gradativa da área da lesão nas vacas de todos os grupos. Essa redução foi mais rápida no primeiro mês e mais lenta até a cura. De forma geral, a lesão apresentou-se menos exsudativa e o prurido foi amenizado a partir da primeira semana de tratamento. Na sequência da evolução as crostas desapareceram e o aspecto ulcerado foi substituído por uma superfície plana de tecido de granulação de cor rosa (Figura 1). O formato circular, presente na grande maioria das vacas estudadas, foi mantido por causa da redução centrípeta da área da ferida. Finalmente, formou-se uma cicatriz de tamanho reduzido.

A escolha dos protocolos de tratamento estudados foi pautada principalmente na segurança em não utilizar um medicamento que pudesse provocar o aparecimento de resíduos no leite. Tratamentos parenterais com ivermectina (GILL et al., 1991) ou com levamisol (RAI et al., 1994) em dose única são apontados como relativamente eficazes, apesar de não produzirem a cura em todos os casos. Entretanto, esses princípios anti-helmínticos não devem ser administrados em vacas durante a lactação (AYRES; ALMEIDA, 2006). É importante destacar que, exceto duas, todas as vacas estudadas estavam em lactação.

Outro critério importante para a escolha dos protocolos de tratamento foi a praticidade e facilidade de execução. Os produtos contendo ivermectina em pasta $1 \%$ e $2 \%$ são disponíveis comercialmente para o tratamento anti-helmíntico de equídeos. A aquisição e a aplicação são fáceis e não há riscos para quem realiza a sua manipulação. Em virtude da proximidade entre a ferida e o tecido mamário pode-se admitir que exista algum risco de difusão da ivermectina e consequente resíduo no leite, mas essa hipótese precisa ser confirmada. Ainda que haja uma pequena distribuição no organismo, esse princípio ativo é marcantemente eliminado pelas fezes (até 98\%) e muito pouco pela urina e pelo leite (até 5\%) (AYRES; ALMEIDA, 2006). Apesar do tratamento ter sido prolongado, a quantidade de pasta utilizada em cada aplicação não excedeu $0,5 \mathrm{~g}$. Considerando-se um peso vivo médio de $400 \mathrm{~kg}$ por vaca, se todo o fármaco fosse absorvido por difusão isso equivaleria a uma dose aproximada de 12 e $25 \mu \mathrm{g} / \mathrm{kg}$ (10 a 20 vezes menor do que a dose anti-helmíntica recomendada).

A concentração de $6 \%$ do triclorfon foi definida com base nos melhores resultados para o tratamento da Stephanofilaria assamensis (SRIVASTAVA; MALVIYA, 1968). A preparação da mistura do triclorfon em pó com a vaselina sólida é muito fácil, pois o triclorfon é altamente lipossolúvel. Em razão dessa propriedade a mistura deve ser manipulada com luva evitando-se o contato com a pele para impedir que a pessoa que executa o tratamento absorva o fármaco. Espera-se, da mesma forma, que ocorra na vaca uma difusão e distribuição após a aplicação na ferida. Entretanto, ainda que todo o fármaco fosse absorvido, a quantidade seria insuficiente para causar efeitos tóxicos. Resíduos no leite não ocorrem com esse princípio ativo (AYRES; ALMEIDA, 2006), o que representa outra vantagem importante.

Acreditando-se que a associação do triclorfon com a ivermectina pudesse produzir um resultado superior, idealizou-se o G4. Optou-se por um produto comercial à base de ivermectina, facilmente utilizado e aprovado para uso em vacas durante a lactação, sem a necessidade de período de carência para a comercialização do leite. Esse tratamento não se provou mais vantajoso. 
Figura 1. Evolução da lesão cutânea causada por Stephanofilaria sp. em uma vaca leiteira, em diferentes momentos ao longo do tratamento tópico com triclorfon a $6 \% \mathrm{em}$ pasta de vaselina. Início do tratamento (A); na primeira semana (B); na terceira semana (C); e cicatrizada na quinta semana (D).

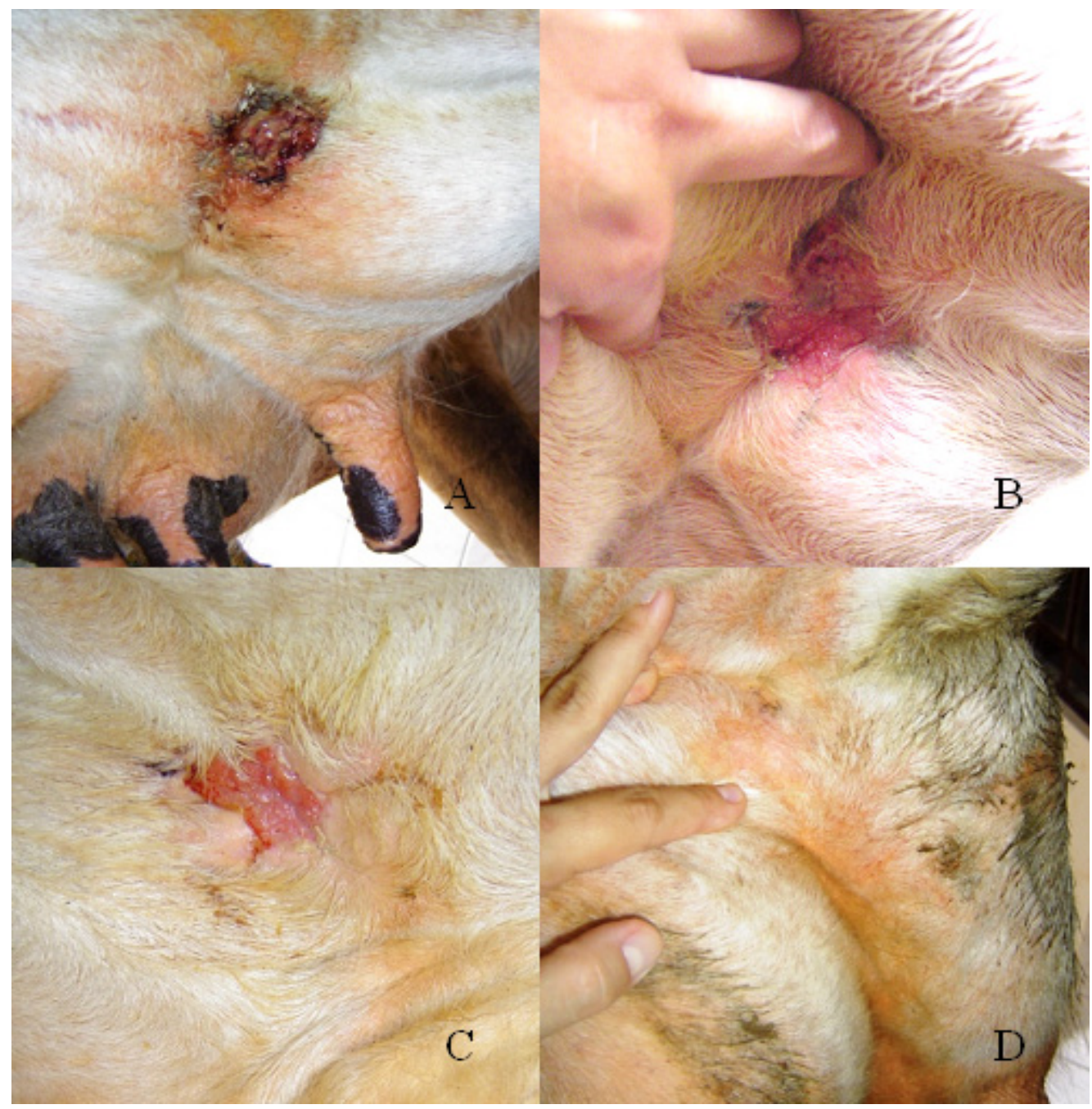

Admitindo o tempo médio em dias para a conclusão de cada tratamento (Tabela 1) e a utilização de $0,5 \mathrm{~g}$ de pasta em cada aplicação, os custos totais puderam ser calculados tomandose como base os preços dos produtos em março de 2011. O custo estimado para a realização do tratamento completo em um animal variou entre os grupos, a saber: $\mathrm{R} \$ 22,00$ para o $\mathrm{G} 1, \mathrm{R} \$ 43,00$ para o G2, R\$4,20 para o G3 e R\$11,00 para o G4.

\section{Conclusão}

Pode-se concluir que a aplicação tópica de triclorfon $6 \%$ em pasta de vaselina, duas vezes ao dia na lesão cutânea é o protocolo mais vantajoso para o tratamento da estefanofilariose em vacas leiteiras, por apresentar a melhor relação custobenefício.

O protocolo experimental foi aprovado pelo Comitê de Ética em Experimentação Animal da UEL (CEEA/UEL) sob o número de registro 61/06. 


\section{Referências}

AYRES, M. C. C.; ALMEIDA, M. A. O. Considerações gerais sobre os anti-helmínticos. In: SPINOSA, $\mathrm{H}$. S.; GÓRNIAK, S. L.; BERNARDI, M. M. (Ed.). Farmacologia aplicada à medicina veterinária. 4. ed. Rio de Janeiro: Guanabara Koogan, 2006. p. 519-526.

BIRGEL, E. H.; PEREIRA, P. C.; MEIRELLES, C. O. S.; AMARAL, V.; ARAÚJO, L. M. Úlcera da lactação filariose provavelmente determinada por Stephanofilaria. Atualidades Veterinárias, v. 1, n. 2, p. 56, 1972.

GILL, B. S.; BALAKRISHNAM, P.; LUMSDEN, G. G.; JONES, P. G. H. Treatment of stephanofilariasis ("earsore") with ivermectin. Veterinary Parasitology, Amsterdam, v. 40, n. 1/2, p. 159-163, 1991.

JOHNSON, S. J.; PARKER, R. J.; NORTON, J. H.; JAQUES, P. A.; GRIMSHAW, A. A. Stephanofilariasis in cattle. Australian Veterinary Journal, New South Wales, v. 57, n. 9, p. 411-413, 1981.

MIYAKAWA, V. I.; REIS, A. C. F.; LISBÔA, J. A. N. Aspectos epidemiológicos e clínicos da estefanofilariose em vacas leiteiras e comparação entre métodos de diagnóstico. Pesquisa Veterinária Brasileira, Brasília,v. 29, n. 11, p. 887-893, 2009.

NOVAES, A. P.; COSTA, A. J. S.; BARBOSA, R. T.; MOREIRA, D. P.; RUZZA, F. J. Dermatite ulcerosa em bovinos provocada por Stephanofilaria. Pesquisa Agropecuária Brasileira, Brasília, v. 3, n. 8, p. 927-929, 1988.

NOVAES, A. P.; OLIVEIRA, M. C. S.; MOREIRA, D. P. Stephanofilaria sp. associada a casos de pododermatite em bovinos leiteiros. Brazilian Archives of Biology and Technology, v. 33, n. 3, p. 575-579, 1990.

OBA, M. S. P.; SINHORINI, I. L.; NOVAES, A. P.; COSTA, A. J. S.; PORTO, A. D. Stephanofilaria em bovinos do município de São Carlos, Estado de São Paulo. In: CONFERÊNCIA ANUAL DA SOCIEDADE PAULISTA DE MEDICINA VETERINÁRIA, 32., 1977, Pirassununga. Anais... Pirassununga: SPMV, 1977. p. 29.
PATNAIK, B. Studies on stephanofilariasis in Orissa: V. treatment and control of "humpsore" in cattle due to Stephanofilaria assamensis. Indian Journal Animal Science, New Delhi, v. 40, n. 2, p. 167-174, 1970.

RAHMAN, A.; KHALEQUE, A. Tratamiento de la filariasis cutânea por Stephanofilaria assamensis "hunpsore" con Neguvón ${ }^{\circledR}$ en el ganado local de Bangladesh. Veterinary Medical Review, v. 4, p. 387-390, 1974.

RAI, R. B.; AHLAWAT, S. P. S.; SINGH, S.; NAGARAJAN, V. Levamisole hydrochloride: an effective treatment for stephanofilarial dermatitis (humpsore) in cattle. Tropical Animal Health and Production, Berlin, v. 26, n. 3, p. 175-176, 1994.

SCOTT, D. W. Parasitic diseases. In: (Ed.). Large animal dermatology. Philadelphia: $\overline{\mathrm{W} . \mathrm{B} \text {. Saunders }}$ Company, 1988. p. 207-283.

SILVA, L. A. F.; BRAGA, C. A.; FIORAVANTE, M. C. S. Estefanofilariose em úbere de vacas lactantes: uma proposta de tratamento. In: CONGRESSO BRASILEIRO DE MEDICINA VETERINÁRIA, 28., 2001, Salvador. Anais... Salvador: [s.n.], 2001. p. 109.

SILVA, L. A. F.; RABELO, R. E.; MOURA, M. I.; FIORAVANTE, M. C. S.; BORGES, L. M. F.; LIMA, C. R. O. Epidemiological aspects and treatment of parasitic lesions similar to Stephanofilariasis disease in nursing cows. Semina: Ciências Agrárias. Londrina, v. 31, n. 3, p. 689-698, 2010.

SRIVASTAVA, H. D.; MALVIYA, H. C. Treatment of "humpsore" in cattle caused by Stephanofilaria assamensis. The Indian Veterinary Journal, Chennai, v. 45, n. 8, p. 484-488, 1968.

UENO, H.; GONÇALVES, P. C. Deteç̧ão dos nematódeos no tecido, por biópsia. In: (Ed.). Manual para diagnóstico das helmintoses de ruminantes. 4. ed. Tokyo: Japan International Cooperation Agency, 1998. p. 105-106. 
